\title{
Journal of

\section{Micro and nanopatterning of functional materials on flexible plastic substrates via site-selective surface modification using oxygen plasma $\dagger$}

\author{
Antony George, Tomasz M. Stawski, Sandeep Unnikrishnan, $\$$ Sjoerd A. Veldhuis and Johan E. ten Elshof* \\ Received 1st October 2011, Accepted 9th November 2011 \\ DOI: 10.1039/c1jm14931h
}

\begin{abstract}
A simple and cost effective methodology for large area micro and nanopatterning of a wide range of functional materials on flexible substrates is presented. A hydrophobic-hydrophilic chemical contrast was patterned on surfaces of various flexible plastic substrates using molds and shadow masks with which selected areas of the substrate surface were shielded from exposure to oxygen plasma. The exposed areas became hydrophilic and were used as templates for site-selective adsorption, electroless deposition and solution phase deposition of functional materials like $\mathrm{ZnO}$, Ag thin films, Au nanoparticles, conducting polymers, titania and $\mathrm{ZnO}$ nanowires. The patterned surfaces and functional materials were characterized by scanning electron microscopy, X-ray diffraction and atomic force microscopy.
\end{abstract}

\section{Introduction}

Flexible polymeric substrates are important in novel roll-to-roll manufacturing technologies. Plastic substrates are ideal candidates for ultra-light electronic devices, personal health monitors and ultrathin foldable display screens due to their flexibility, low weight and inexpensiveness. In order to realize functional flexible devices, the ability to pattern various materials such as conducting polymers, metals and metal oxides in the form of thin films, nanoparticle assemblies, or nanowire arrays is crucial. To ensure high yield and low cost production, the availability of cost-effective, low temperature patterning processes under mild chemical conditions is required. Conventional device fabrication approaches such as photolithography, chemical vapor deposition or wet/dry etching often require a clean room and are not always compatible with plastic substrates due to the instability of organic polymers in such process environments.

Recent methodologies such as surface modification, ${ }^{1}$ material transfer $^{2}$ and self-assembly ${ }^{3}$ are ideal to combine with plastic substrates, due to their technical simplicity and mild processing conditions. Site-selective surface modification of flexible substrates,

MESA+ Institute for Nanotechnology, University of Twente, P.O. Box 217, 7500 AE Enschede, The Netherlands.E-mail: j.e.tenelshof@utwente. $n l$

$\dagger$ Electronic supplementary information (ESI) available: Experimental section. See DOI: $10.1039 / \mathrm{cljm} 14931 \mathrm{~h}$

\$ Present address: TNO Holst Centre, Eindhoven, the Netherlands followed by local immobilization or growth of materials is an ideal strategy for patterning functional materials on flexible substrates. In the present paper we employed different templates such as soft lithographic polydimethylsiloxane (PDMS) molds and silicon-based micro/nano stencil shadow masks to pattern flexible substrates like PDMS, polycarbonate (PC) and polyethylene terephtalate (PET) site-selectively using oxygen plasma. The patterned substrates are used for area-selective deposition of functional polymeric, metallic and ceramic materials.

A schematic diagram of the patterning process is shown in Fig. 1a. First conformal contact is made between the shielding template, i.e., a patterned soft lithographic PDMS mold with connected channel structure or a silicon-based shadow mask, and the flexible polymeric substrate. The substrate-template assembly is then exposed to oxygen plasma. The plasma diffuses and reaches all non-shielded areas where it oxidizes the substrate surface, so that it becomes polar and hydrophilic. The regions on the substrate that are in conformal contact with the protruding parts of the PDMS mold or the shadow mask are protected from surface modification and remain hydrophobic.

After the prepatterning step, functional materials are selectively patterned onto the hydrophilic areas via electroless deposition, siteselective de-wetting, colloidal self-assembly and/or solution phase deposition. We demonstrate the versatility of our approach by patterning a wide range of functional materials such as $\mathrm{ZnO}, \mathrm{ZnO}$

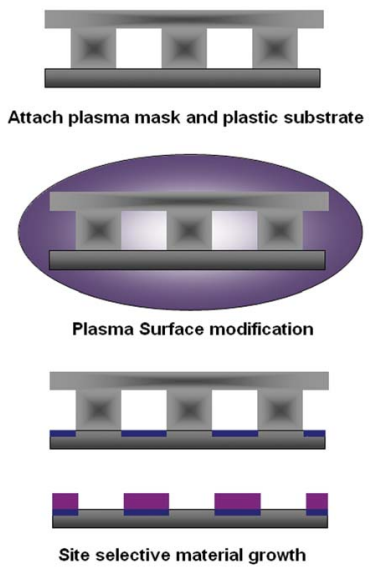

Fig. 1 Schematic diagram of the channel diffused plasma patterning process and plasma modification. 
nanowires, $\mathrm{TiO}_{2}$, silver, gold nanoparticles and the conducting polymer poly(2,3-dihydrothieno-1,4-dioxin)-poly(styrene sulfonate) (PEDOT:PSS) on length scales down to a few hundred nanometres.

\section{Results and discussion}

Oxygen plasma treatment is commonly used as a technique to clean substrates ${ }^{4,5,6}$ and chemically modify surfaces to promote the wetting properties. ${ }^{4,7}$ In soft lithographic approaches like micromolding in capillaries (MIMIC) and microtransfer molding ( $\mu \mathrm{TM}$ ), oxygen plasma is used to increase the surface energy of the PDMS surface to improve the wetting and/or promote the flow of sol-gel precursor solutions in PDMS channels. ${ }^{5,6,7}$ Exposure of plastic surfaces such as PET, PC, and poly-methyl-methacrylate (PMMA) to oxygen plasmas creates surface $-\mathrm{CO}$ and $-\mathrm{COOH}$ groups which are responsible for the increased hydrophilicity of the exposed surface. ${ }^{8,9,10}$ Also slight etching and roughening was observed on the polymeric PET and PC substrates due to the exposure to oxygen plasma. However, the etch rates were low as $0.5 \mathrm{~nm} \mathrm{~min}{ }^{-1}$. In the case of PDMS, plasma exposure yields a silica $\left(\mathrm{SiO}_{\mathrm{x}}\right)$-like brittle surface. ${ }^{11}$ All surfaces returned to their previous hydrophobic state in the course of time after exposure. ${ }^{\mathbf{8 , 9 , 1 1}}$

The inset of Fig. 2a shows an HR-SEM image of a microstencil that we used for our experiments and Fig. 2a itself shows an HRSEM image of a plasma-patterned PDMS substrate using the same microstencil. The stencil has perforations of $5 \mu \mathrm{m}$; the oxidized dots on the PDMS substrate have the same diameter. Fig. $2 b$ shows plasma-patterned PDMS substrates using nanostencils with $400 \mathrm{~nm}$ perforations. Nanostencil lithography was only possible on PDMS substrates since the elasticity of the PDMS allowed to make good conformal contact between stencil and substrate. On other substrates such as PC and PET we used micro and nanopatterned PDMS molds to guide the oxygen plasma inside the channels between substrate and mold to form a chemically contrasted prepatterned substrate onto which complex oxide patterns were formed in a subsequent deposition step. The plasma diffusion process inside the PDMS channels is time and geometry-dependent. ${ }^{12,13}$ A substantial degree of chemical

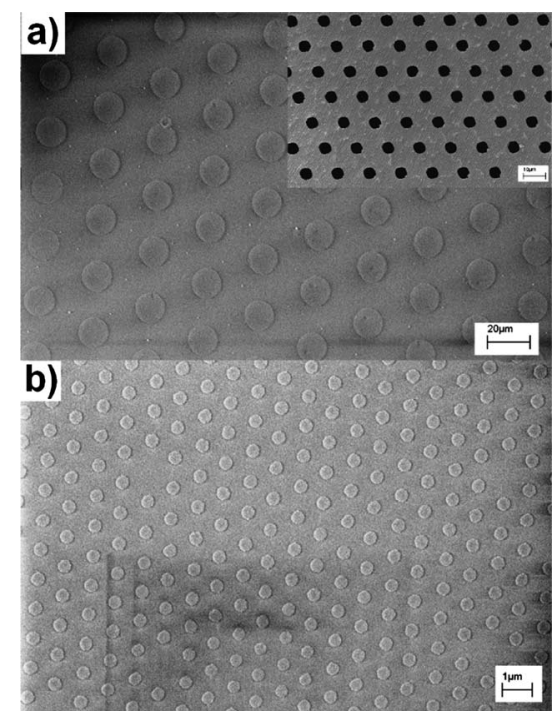

Fig. 2 a) HR-SEM images of PDMS substrate after plasma patterning using microstencil with $5 \mu \mathrm{m}$ perforations shown in the inset; b) PDMS substrates with $400 \mathrm{~nm}$ wide oxidized dots. modification could be achieved by plasma exposure over an area of $1 \times 1 \mathrm{~cm}^{2}$ within $10 \mathrm{~min}$ of exposure. The same molds could be reused for many patterning cycles without loss of quality of the replicated patterns. We tested different PDMS mold pattern geometries, including straight lines and interconnected network-type channel structures such as square grids, circular grids and honeycomb-type channel arrays. Optical microscopy examination confirmed that surface modification occurs faster when connected channel structures (circular grid, square grid, honeycomb patterns, etc.) were used, when compared to pattern geometries derived from non-connected channel structures (straight lines, zigzag lines, etc.). Modification times of up to $15-20 \mathrm{~min}$ were required to modify $8 \mathrm{~mm}$ long straight channels. We also examined PDMS molds with different height-to-width aspect ratios to determine the dependence of the plasma diffusion rate on aspect ratio. PDMS channels with a higher aspect ratio allowed faster diffusion of plasma, so that substantial diffusion distances could be accomplished in less time. The results are in agreement with a similar strategy, reported by Langowski et al., to prepattern a hydrophobic-hydrophilic contrast prior to deposition of biomolecular inks. ${ }^{1}$

$\mathrm{ZnO}$ thin films were deposited on plasma patterned silver seeded regions of the substrate following the recipe of Izaki and Omi. ${ }^{14}$ The film growth mechanism is explained in detail in ref. [14]. First a silver seeding step was employed to attach silver ions to the oxidized areas of the substrate and followed by a photoreduction step to convert the silver ions to silver particles. The silver particles acted as nucleation sites for $\mathrm{ZnO}$ growth. Fig. 3a shows a zigzag line pattern of $\mathrm{ZnO}$ formed on plasma modified silver seeded regions of a PET substrate. Fig. 3b show high aspect ratio honeycomb patterns of $\mathrm{ZnO}$ grown on a PDMS substrate. The hydrophobic non-seeded regions of the substrates were almost free from $\mathrm{ZnO}$. However, a few small particles of $\mathrm{ZnO}$ were found in the non-seeded areas. These were attributed to homogeneously nucleated particles from the growth solution. To illustrate the versatility of the technique we used different pattern geometries, including lines, dots, grids and honeycomb patterns. The SEM images in Fig. 3 show that nucleation took place within the plasma modified and seeded regions of the substrate.

Fig. $3 \mathrm{c}$ show the tapping mode AFM height profile of high aspect ratio $\mathrm{ZnO}$ pattern on PDMS substrate, corresponding to the SEM images of Fig. 3b. A $\mathrm{ZnO}$ film of $\sim 2 \mu \mathrm{m}$ thickness formed on the plasma-modified silver seeded areas after $5 \mathrm{~h}$ of deposition time. The wurtzite phase of $\mathrm{ZnO}$ was confirmed by the XRD diffractogram in Fig. 3d. The rate of deposition was approximately 5-6 $\mathrm{nm} \mathrm{min} \mathrm{min}^{-1}$, independent of deposition time. However, the SEM images showed that during the initial 15-30 min of film growth the film density was not uniform. Longer deposition times yielded more uniformly covered films.

Fig. 3e and $3 \mathrm{f}$ shows SEM images of $\mathrm{ZnO}$ nanowires deposited on plasma-patterned regions of the PDMS substrate. The $\mathrm{ZnO}$ nanowires were grown according to the method of Greene et al. ${ }^{15}$ First, a $\mathrm{ZnO}$ seed growth step was performed by immersing the plasma patterned substrates in a $0.5 \mathrm{~mol} \mathrm{dm} \mathrm{dm}^{-3}$ ethanolic solution of zinc acetate, followed by drying at $80{ }^{\circ} \mathrm{C}$ for $1 \mathrm{~h}$. A subsequent oxygen plasma treatment was employed to convert the zinc acetate phase to $\mathrm{ZnO}$ seed particles. Then the substrates were kept floating on the surface of the nutrient solution, with the $\mathrm{ZnO}$-seeded side facing downward into the solution at $80^{\circ} \mathrm{C}$. This was essential to avoid any deposition of homogeneously nucleated material on the substrate. 

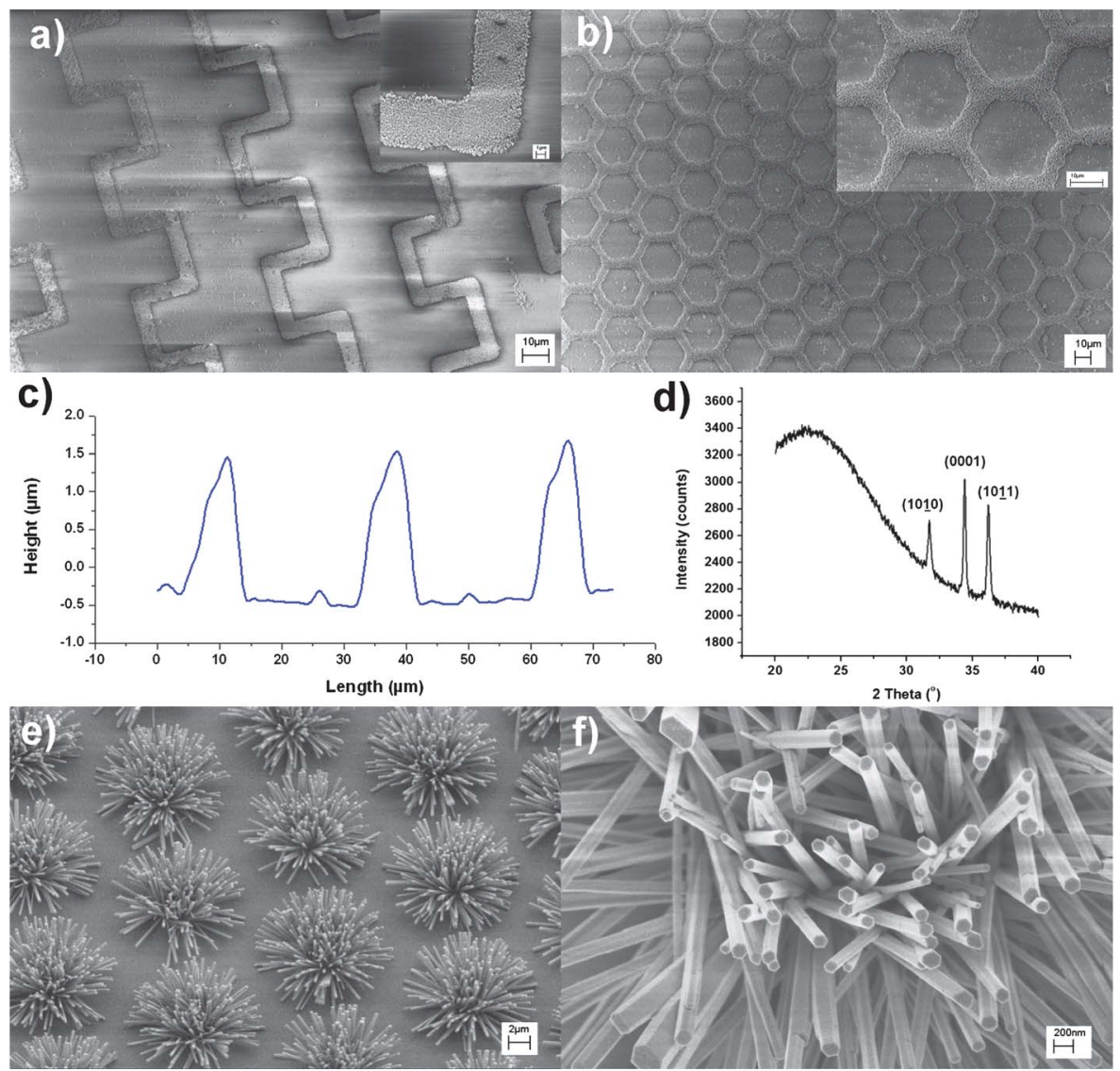

Fig. 3 SEM images of $\mathrm{ZnO}$ patterns formed by area-selective electroless deposition on plasma-modified Ag-seeded regions on PDMS and PET substrates. a) Zigzag line pattern of $\mathrm{ZnO}$ on PET substrate, line width $5.3 \mu \mathrm{m}$; spacing $2.8 \mu \mathrm{m}$; b) High aspect ratio honeycomb pattern on PDMS substrate, line width $3.2 \mu \mathrm{m}$; c) Tapping mode height profile of high aspect ratio $\mathrm{ZnO}$ pattern shown in Figure $3 \mathrm{~b}$. The patterns grew to $\sim 2 \mu \mathrm{m}$ thickness in $5 \mathrm{~h}$ time; d) XRD spectrum of $\mathrm{ZnO}$ thin films grown at $55^{\circ} \mathrm{C}$. e-f) SEM images of $\mathrm{ZnO}$ nanowires grown on channel diffused plasma modified regions of PDMS substrate at different magnifications. Growth time was $15 \mathrm{~h}$. The nanowires were $\sim 3 \mu \mathrm{m}$ long.

The $\mathrm{ZnO}$ nanowires grew downwards, following the crystal orientation of the $\mathrm{ZnO}$ seeds. The XRD data confirmed the preferential [0001] growth direction of wurtzite $\mathrm{ZnO}$. The growth rate of the nanowires was $\sim 100-200 \mathrm{~nm} \mathrm{~h}^{-1}$. We grew nanowires for periods of 1 to $24 \mathrm{~h}$. In view of the considerable reduction of $\mathrm{Zn}^{2+}$ ion concentration in the solution due to consumption by growth, it was necessary to replace the nutrient solution after every $10 \mathrm{~h}$ to maintain a constant growth rate.

Fig. 4a shows titania patterns formed on a PET substrate. A tapping mode height profile of amorphous titania pattern are shown in Fig. 4b. The formation of titania was confirmed by EDX analysis. The patterns have an average height of $80 \mathrm{~nm}$ and a surface roughness of $\sim 30 \mathrm{~nm}$. Patterns of amorphous $\mathrm{TiO}_{2}$ were obtained by a selective deposition and subsequent hydrolysis of titanium(Iv) phenylmethoxide. The precursor was synthesized by reacting titanium(Iv) isopropoxide with benzyl alcohol yielding a $0.2 \mathrm{~mol} \mathrm{dm}^{-3}$ solution. The ${ }^{1} \mathrm{H}$ NMR measurements showed that at least $88 \mathrm{~mol} \%$ of the isopropoxy ligands were directly exchanged by benzyl alcohol ligands during preparation. ${ }^{16}$ By this means the overall reactivity of titanium(Iv) precursor was significantly decreased, so that it could have been handled relatively easily in a typical laboratory environment. Sol-gel processing of titanium(Iv) alkoxide into titanium oxide gel follows through hydrolysis and condensation reactions. ${ }^{17}$
Depending on hydrolysis ratio, used catalysts, and coordinating alcoholic ligands, different titania morphologies can be obtained. ${ }^{18}$ The as-prepared titania is amorphous, but can be converted easily into crystalline rutile or anatase by mild hydrothermal treatment. ${ }^{19}$

Metallic patterns on plastic substrates are important due to their application as interconnects and active components like sensing elements in flexible devices. We used silver-seeded plasma-treated regions of the plastic substrates for site-selective electroless deposition of Ag. Fig. $4 \mathrm{c}$ and $4 \mathrm{~d}$ show an HR-SEM image, and a tapping mode AFM height profile of Ag micropatterns formed over plasmapatterned silver-seeded PC substrate. The patterns have a line width of $8 \mu \mathrm{m}$ and a line spacing of $1.2 \mu \mathrm{m}$. The deposition time was $15 \mathrm{~min}$ for a thickness of $150 \mathrm{~nm}$. The growth rate can be controlled by dilution of the growth solution, and film thickness can be controlled by deposition time.

Conducting polymers such as PEDOT:PSS and polyaniline (PANI) are important materials in the manufacture of flexible devices such as flexible displays. Fig. 5a-c shows an HR-SEM image, a tapping mode AFM 3D topography image and an AFM height profile of PEDOT:PSS patterns spin-coated over a plasma-patterned PDMS substrate made with a silicon stencil. The dot patterns have a diameter of $5 \mu \mathrm{m}$ and a spacing of $5 \mu \mathrm{m}$ between the dots. The height of each PEDOT:PSS dot is $65 \mathrm{~nm}$. 

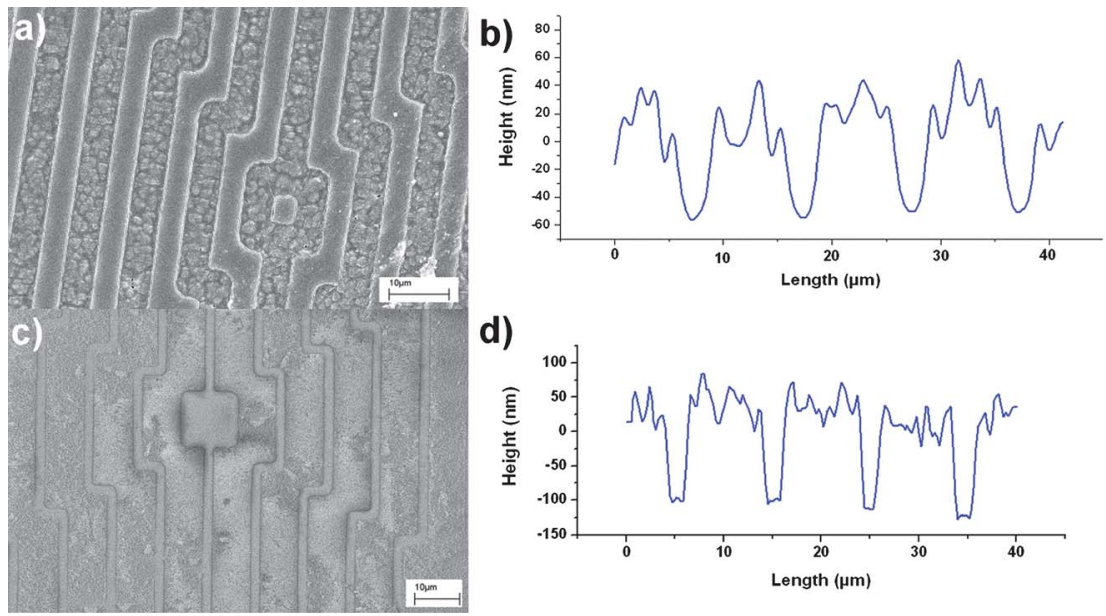

d)

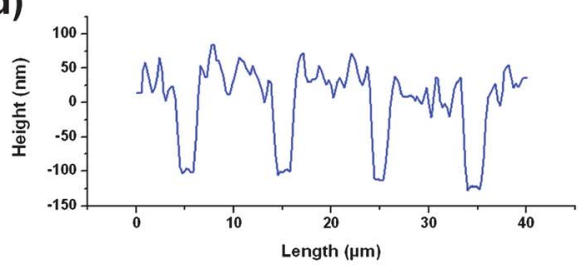

Fig. 4 a) HR-SEM images of $\mathrm{TiO}_{2}$ patterns on PET substrate. b) Tapping mode AFM height profile of TiO2 pattern; c) HR-SEM images of Ag micropatterns on PC substrate; d) Tapping mode AFM height profile of Ag pattern grown on PC substrate.
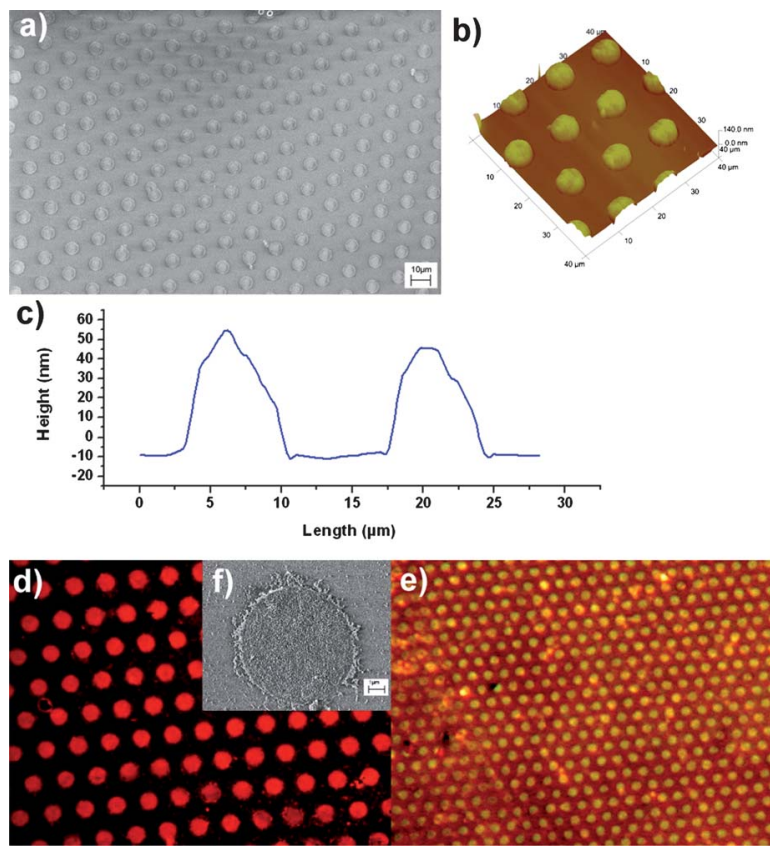

Fig. 5 a) HR-SEM image of PEDOT:PSS dot array selectively spincoated on the hydrophilic regions of the substrate. b) Tapping mode AFM 3D topographic image of PEDOT:PSS dots. c) AFM height profile of PEDOT:PSS dots. d) Optical microscopic image of Au nanoparticle arrays of $5 \mu \mathrm{m}$ dots selectively adsorbed on the stencil patterned substrates; e) optical micrograph of $800 \mathrm{~nm}$ dots; f) SEM image of a $5 \mu \mathrm{m}$ dot with adsorbed Au nanoparticles.

Fig. 5d and 5e show optical microscope images and Fig. 5f shows HR-SEM image of Au nanoparticles that were selectively attached on an APTES-patterned PDMS substrate. After plasma modification on PDMS substrates using micro and nanostencils, APTES molecules were selectively bonded to the modified areas. The hydroxyl groups on the oxidized areas of the substrate reacted with APTES and formed a self-assembled molecular thin film. Gold nanoparticles with an average size of $13-15 \mathrm{~nm}$ were deposited selectively on the aminosilane areas by immersing the patterned substrates in a colloidal solution of gold nanoparticles. The $-\mathrm{C}_{3} \mathrm{H}_{6} \mathrm{NH}_{2}$ side chains in the film were protonated at a $\mathrm{pH}$ of 2.5 and formed $-\mathrm{NH}_{3}{ }^{+}$end groups which selectively bonded to negatively charged citrate ioncoated $\mathrm{Au}$ nanoparticles.

\section{Conclusions}

We have demonstrated a fast and easy approach to pattern a wide range of functional materials, including $\mathrm{ZnO}, \mathrm{ZnO}$ nanowires, $\mathrm{Au}$ nanoparticles, $\mathrm{Ag}$ and PEDOT:PSS on flexible substrates like PDMS, PC and PET by oxygen plasma-modified patterning using PDMS molds or micro/nanostencil shadow masks. We made patterns of arbitrary complexity, with different geometrical features such as lines, anitidots, honey comb and isolated dot arrays. The patterns formed by this technique have feature dimensions as small as $400 \mathrm{~nm}$, up to a few micrometres and cover several square millimetres without defects. Plasma-patterned surface modification using shielding templates is a simple, cost effective and efficient technique to pre-pattern plastic substrates for subsequent site-controlled deposition of metal oxides, metals and organic materials via a range of methods, e.g. electroless deposition, solution phase deposition, siteselective adsorption and site-selective de-wetting.

\section{Acknowledgements}

Financial support of NWO-STW in the framework of the Innovational Research Incentive (VIDI scheme) is acknowledged.

\section{References}

1 B. A. Langowski and K. E. Uhrich, Langmuir, 2005, 21, 10509.

2 Y. Yang, Y. Hwang, H. A. Cho, J.-H. Song, S.-J. Park, J. A. Rogers and H. C. Ko, Small, 2011, 7, 484.

3 S. S. Kim, E. Saeedi, J. R. Etzkorn and B. A. Parviz, in Automation Science and Engineering, 2008. CASE 2008. IEEE International Conference, 2008, pp. 967-970.

4 S. U. Khan, O. F. Gobel, D. H. A. Blank and J. E. ten Elshof, $A C S$ Appl. Mater. Interfaces, 2009, 1, 2250.

5 O. F. Gobel, D. H. A. Blank and J. E. ten Elshof, ACS Appl. Mater. Interfaces, 2010, 2, 536.

6 J. E. ten Elshof, S. U. Khan and O. F. Göbel, J. Eur. Ceram. Soc., 2010, 30, 1555. 
7 A. George, A. W. Maijenburg, M. G. Maas, D. H. A. Blank and J. E. ten Elshof, ACS Appl. Mater. Interfaces, 2011, 3, 3666.

8 J. Abenojar, R. Torregrosa-Coque, M. A. Martinez and J. M. MartinMartinez, Surf. Coat. Technol., 2009, 203, 2173.

9 A. Vesel, M. Mozetic and A. Zalar, Vacuum, 2007, 82, 248.

10 D. Xia, X. He, Y.-B. Jiang, G. P. Lopez and S. R. J. Brueck, Langmuir, 2010, 26, 2700.

11 M. K. Chaudhury and G. M. Whitesides, Langmuir, 1991, 7, 1013.

12 M.-H. Lin, C.-F. Chen, H.-W. Shiu, C.-H. Chen and S. Gwo, J. Am. Chem. Soc., 2009, 131, 10984.

13 A. George, A. W. Maijenburg, M. G. Maas, D. H. A. Blank and J. E. ten Elshof, Langmuir, 2011, 27, 12235.
14 M. Izaki and T. Omi, J. Electrochem. Soc., 1997, 144, L3.

15 L. E. Greene, M. Law, J. Goldberger, F. Kim, J. C. Johnson, Y. Zhang, R. J. Saykally and P. Yang, Angew. Chem., Int. Ed., 2003, 42, 3031.

16 T. M. Stawski, S. A. Veldhuis, O. F. Göbel, J. E. ten Elshof and D. H. A. Blank, J. Am. Ceram. Soc., 2010, 93, 3443.

17 J. D. Wright and N. A. J. M. Sommerdijk, "Sol-gel Materials Chemistry and Applications", Advanced Chemistry Texts, CRC Press, Boca Raton, 2001, 53-68.

18 U. Schubert, J. Mater. Chem., 2005, 15, 3701.

19 C.-C. Wang and J. Y. Ying, Chem. Mater., 1999, 11, 3113 . 